\title{
Distributed Admission Control in Multiservice IP Networks: Concurrency Issues
}

\author{
Solange Rito Lima, Paulo Carvalho, and Vasco Freitas \\ University of Minho \\ Department of Informatics \\ 4710-057 Braga, Portugal \\ Email: $\{$ solange,pmc,vf $\} @$ di.uminho.pt
}

\begin{abstract}
In distributed admission control (AC) schemes, handling concurrent AC decisions assumes a relevant role in avoiding over or false acceptance and, consequently, service quality degradation. This problematic is even more intricate in multiservice network environments where distinct service levels need to be fulfilled. This paper debates and points out solutions to mitigate the negative impact that distributed admission of flows might have on the service level guarantees provided to network customers. Keeping in mind that simplicity is a key factor for deployable AC solutions, we suggest and discuss the use of (i) a service-dependent concurrency index; (ii) a token-based system and (iii) a rate-based credit system, as alternative or complementary proposals to minimize or solve QoS degradation resulting from $\mathrm{AC}$ false acceptance.
\end{abstract}

Index Terms-Concurrent Admission Control; Quality of Service; Differentiated Services; Multiservice Networks

\section{INTRODUCTION}

Supporting QoS in the Internet launches new demands and challenges on the provision and management of multiservice networks and underlying resources. To guarantee that QoS commitments are honored, traffic control mechanisms such as admission control (AC) are recommended for keeping service classes under controlled load and assuring QoS levels [1]-[3]. Despite this need, a major objective to keep in mind, and likely a key aspect for their deployment in real networks, is to maintain the network control plane complexity as low as possible. In this context, a lightweight and distributed AC model based on on-line QoS monitoring feedback for managing multiple services quality has been proposed in [4] and formalized in [5]. This model allows to control QoS and the utilization of Service Specification Levels (SLSs) both intra and interdomain.

Distributed AC models, by their nature, are likely to involve multiple and simultaneous AC decisions. Therefore, the need for handling concurrency, which stems

Based on "Handling Concurrent Admission Control in Multiservice IP Networks" by S. Rito Lima, P. Carvalho, and V. Freitas which appeared in the Proceedings of the IEEE Consumer Communications and Networking Conference (CCNC 2006), Las Vegas, U.S.A., January 2006. (C2006 IEEE. from having multiple decision points, is justified as a way to avoid over/false acceptance of flows entering the network causing, consequently, resources overload and service degradation. This degradation, more likely to occur when the number of ingress nodes accepting incoming calls increases, may be unacceptable for QoS demanding services such as IP telephony.

Although distributed AC has been matter of intense study (see Section II), the problem of concurrent AC in multiservice networks has been rarely tackled. In [6], a given amount of bandwidth, called AC Limit, is defined as a reference value for the acceptable traffic within a class. AC Limits are defined off-line at an initial provisioning phase taking as input: (i) the network topology (ii) the long-term expected traffic matrices; and (iii) the bandwidth sharing policies among classes. The initial static limits can be extended dynamically by sharing unused AC Limits between egress routers.

This paper extends these concepts and points out several new proposals that may be adopted to control the admission of concurrent flows, so that the service level guarantees negotiated with customers are protected from overacceptance. These alternative or complementary proposals include the definition of:

- a per-class concurrency index;

- a token-based system;

- a rate-based credit system controlled by egress nodes.

In the latter approach, an amount of rate credits is assigned to each ingress node, considering: (i) the negotiated (upstream and downstream) SLSs; (ii) the dynamic negotiation of new SLSs; (iii) the AC of flows when sustained or not by an individual SLS. In this system, the concurrency problem is implicitly considered and minimized as long as each ingress node maintains its flows' acceptance level within the available credits of each service class.

The remaining of this paper is structured as follows: current proposals focusing on distributed AC are debated in Section II; the characteristics of the multiservice $\mathrm{AC}$ model used as case study, the main network domain entities and the $\mathrm{AC}$ criteria are summarized 
in Section III, the study of concurrent AC and the proposals for tackling concurrency shortcomings are debated in Section IV; finally, the conclusions are drawn in Section V.

\section{RELATED WORK}

Common AC approaches for class-based IP networks are either centralized (e.g. based on bandwidth brokers [7], [8]) or distributed, parameter or measurementbased differing on the type of services being supported.

The main advantage of centralized AC approaches is that centralizing state information and control tasks allows a global vision of the domain's QoS and operation, relieving the control plane inside the network. This centralization process also facilitates creating and changing service policies and control mechanisms such as AC algorithms. The cost of centralized approaches is however high. Central entities need to store and manage large amounts of information, which in large and highly dynamic networks with many signaling messages and information state updates needing to be processed in real-time is even hard or prohibitive. The congestion and functional dependence on a single entity is another well-known problem of centralization.

Therefore, to improve reliability and scalability in large network domains, several approaches consider distributed AC with variable control complexity depending on the QoS guarantees and predictability required. To provide guaranteed services (e.g., for hard real-time traffic), AC proposals tend to require significant network state information and, in many cases, changes in all network nodes [9], [10]. To provide predictive services (e.g., for soft real-time traffic) measurementbased AC (MBAC) [11], [12] and end-to-end MBAC (EMBAC) solutions [13]-[15] have deserved special attention. These solutions leads to reduced control information and overhead, but eventually to QoS degradation. To control elastic traffic, for more efficient network utilization, implicit AC strategies, i.e., without requiring explicit signaling between the application and the network, have also been defined [16], [17].

As far as distributed AC is concerned, this paper extends the former studies by focusing on debating the problem of concurrent $\mathrm{AC}$ and putting forward solutions to mitigate the negative impact concurrency might have on the provided service level guarantees. To sustain this debate a distributed AC proposal is presented and summarized next.

\section{AC Model SpecificAtion}

\section{A. Model Overview}

This section provides a brief overview of the multiservice AC model proposed in [4], [5] before debating AC concurrency. This model resorts to edge-to-edge on-line monitoring to obtain feedback of each service class's performance so that proper AC decisions are made. To control dynamically customers' traffic entering a network domain, the model's underlying AC rules control both QoS levels in the domain and the sharing of active SLS between domains.

As illustrated in Fig. 1, while ingress routers perform explicit or implicit AC depending on the application type and corresponding service class, egress routers perform on-line QoS monitoring and SLS control. Online QoS Monitoring, carried out on an ingress-egress basis, measures specific metrics for each service type, providing a quantitative view of the service level available from each ingress node. SLS Control monitors the usage of downstream SLSs at each egress, to ensure that traffic to other domains does not exceed the negotiated profiles and packet drop will not occur due to a simple and indiscriminate traffic conditioning process. The obtained measures are sent to the corresponding ingress routers to update an Ingress-Egress service matrix used for distributed AC and active service management. This notification is carried out periodically, when a metric value or its variation exceeds a limit or the SLS utilization exceeds a safety threshold.

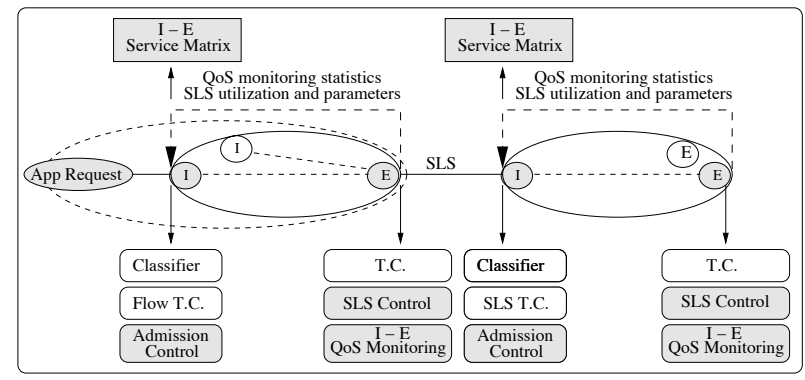

Fig. 1. Location of tasks in a multiclass domain

The end-to-end case, detailed in [5], is viewed as a repetitive and cumulative process of $\mathrm{AC}$ and available service computation only performed at ingress nodes.

As the proposed model is multiservice, explicit and implicit AC can be in place depending on the application or service characteristics. Explicit flow AC is oriented to applications able to signal the network with their traffic profile and QoS objectives. In this case, the $\mathrm{AC}$ decision requires two initial verifications regarding SLS utilization control and QoS control following the rules defined in III-C.

Implicit flow $\mathrm{AC}$, oriented to applications which do not use signaling and in particular to elastic applications, use implicit detection/rejection of flows [16]. In this type of AC, neither specific flow's information nor measures from upstream domains are considered. 


\section{B. Multiservice Domain Specification}

Considering the overview of the AC model operation described above, we focus now the main components of a generic network domain comprising multiple ingress and egress routers, as regards the provision of multiservices to customers (individuals or other domains). Following [5], we specify the following domain entities: (i) service classes; (ii) upstream SLSs; (iii) downstream SLSs and (iv) traffic flows. Network resources are implicitly considered and controlled by the edge-to-edge monitoring process. When possible, the entities under specification use indexes based on the corresponding service class and involved ingress and egress nodes. As the AC model is class-based and operates edge-to-edge, this approach enriches semantically the notation, while keeping it intuitive.

\section{Service Classes}

Considering a multiclass domain $D_{x}$ comprising $N$ ingress nodes and $M$ egress nodes, we define $I^{D_{x}}=\left\{I_{1}, I_{2}, \ldots, I_{N}\right\}$ and $E^{D_{x}}=\left\{E_{1}, E_{2}, \ldots, E_{M}\right\}$ as the set of ingress and egress nodes, respectively ${ }^{1}$. For this domain, we represent the set of supported service classes as $S C^{D_{x}}=\left\{S C_{1}, S C_{2}, \ldots, S C_{Y}\right\}$. For each service class $S C_{i} \in S C^{D_{x}}$, the set of QoS parameters under control is defined as $P_{S C_{i}}=$ $\left\{\left(P_{i, 1}, \beta_{i, 1}\right), \ldots,\left(P_{i, P}, \beta_{i, P}\right)\right\}$ where each $P_{i, p} \in P_{S C_{i}}$ is the class parameter target value and $0 \leq \beta_{i, p} \leq 1$ is the parameter's safety margin. Each parameter's upper bound or threshold, given by $T_{i, p}=\beta_{i, p} P_{i, p}$, is used to trigger $\mathrm{AC}$.

In practice, the service classes to be supported in $D_{x}$ are closely related to the service levels negotiated with both upstream and downstream customers. Thus, $D_{x}$ is a service provider for an upstream domain $D_{x}^{-}$and a customer of a downstream domain $D_{x}^{+}$. Lets now consider that $S L S_{i, I_{n}}$ identifies a specific SLS accepted for $S C_{i}$ with upstream domain $D_{x}^{-}$, connecting $D_{x}$ through $I_{n}$, and $S L S_{i, E_{m}}^{+}$identifies a specific SLS negotiated for $S C_{i}$ with downstream domain $D_{x}^{+}$, accessible from $D_{x}$ through $E_{m}$ (see Fig. 2).

The case of flows entering the domain $D_{x}$ without pre-negotiated SLSs (usually dial-up users) is also covered, and the notation $\notin S L S$ is introduced for this purpose. The global rate share of these users is controlled by $R_{i, I_{n}}^{\notin S L S}$. Therefore, $R_{i, I_{n}}^{\notin S L S}$ is a ratebased parameter defined to limit traffic not sustained by a specific SLS.This allows a better control of the rate share in $D_{x}$ and of $S L S_{i, E_{m}}^{+}$utilization, while avoiding possible denial-of-service to flows belonging to $S L S_{i, I_{n}}$.

\footnotetext{
${ }^{1}$ To simplify the notation, and without losing generality, each ingress or egress distinct interface is treated as a virtually distinct
} ingress $I_{n}$ or egress node $E_{m}$.

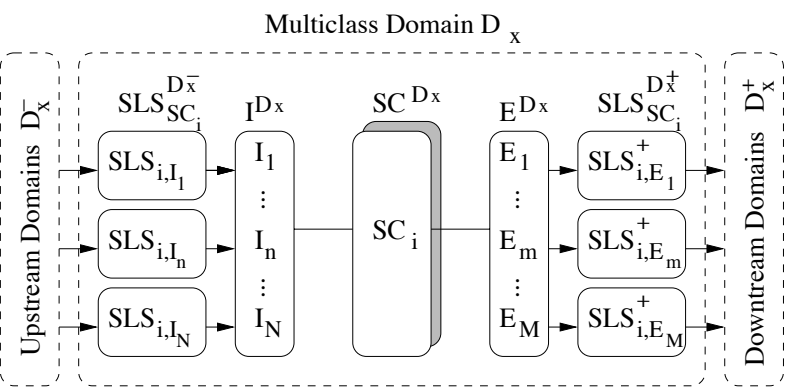

Fig. 2. Domain Elements and Notation

\section{Upstream SLSs}

The definition of SLSs [18], apart from being a key aspect for QoS provisioning, provides a valuable input for AC, in special, when admission spans multiple domains. From an AC perspective, an upstream SLS for service class $S C_{i}$, i.e., $S L S_{i, I_{n}}$, includes elements such as those included in Table I.

TABLE I

COMMON $S L S_{i, I_{n}}$ ELEMENTS

\begin{tabular}{|lll|}
\hline Item & Notation & Example \\
\hline 1) Scope & $S L S_{i, I_{n}} \rightarrow$ Scope & $\left(I_{n}, E^{\prime}\right)$ \\
2) Service ID & $S L S_{i, I_{n}} \rightarrow S C_{i d}$ & $D S C P$ \\
3) Traffic Profile & $S L S_{i, I_{n}} \rightarrow T P r o f$ & $T B\left(R_{i, I_{n}}, b_{i, I_{n}}\right)$ \\
4) Expected QoS & $S L S_{i, I_{n}} \rightarrow$ EQoS & $I P T D_{i, I_{n}}$ \\
5) Validity & $S L S_{i, I_{n}} \rightarrow$ Sched & {$\left[t_{i, I_{n}, 0}, t_{i, I_{n}, f}\right]$} \\
\hline
\end{tabular}

1) $S L S_{i, I_{n}} \rightarrow$ Scope is specified as a pair $\left(I_{n}, E^{\prime}\right)$ where $I_{n}$ is the access point of the upstream domain $D_{x}^{-}$to $D_{x}$ and $E^{\prime} \subseteq E^{D_{x}}$ represents all possible egress nodes $E_{m}$ providing access from $D_{x}$ to $D_{x}^{+}$for this $S L S$. At this point, the scope of $S L S_{i, I_{n}}$ is limited to a single domain $D_{x}$, which is responsible for identifying $E^{\prime}$ according to the destination domains $D_{x}^{+}$defined in $S L S_{i, I_{n}}$.

2) $S L S_{i, I_{n}} \rightarrow S C_{i d}$ identifies the service type to be provided by $D_{x}$ to packets belonging to $S L S_{i, I_{n}}$. The DS Code Point is a possible $S C_{i d}$ in Diffserv domains.

3) $S L S_{i, I_{n}} \rightarrow$ TProf specifies the qualitative and/or quantitative traffic characteristics of $S L S_{i, I_{n}}$, allowing to identify traffic as in or outof-profile. The rate $R_{i, I_{n}}$ represents the global aggregate rate established for $S L S_{i, I_{n}}$ within the scope region.

4) $S L S_{i, I_{n}} \rightarrow E Q o S$ specifies the expected QoS parameters for $S L S_{i, I_{n}}$, i.e., $P_{S L S_{i_{I}}}=$ $\left\{P_{i, I_{n}, 1}, \ldots, P_{i, I_{n}, P^{\prime}}\right\}$. Each QoS parameter $P_{i, I_{n}, p}$ value is bounded by the corresponding service class $P_{i, p}$, regardless the incoming $I_{n}$ and accepted $S L S_{i, I_{n}}$. Embedding the expected SLS 
parameters values in the respective class parameter target values is of paramount importance as QoS and SLS control in the domain is clearly simplified. Examples of $P_{i, I_{n}, p}$ are $I P T D_{i, I_{n}}$, $i p d v_{i, I_{n}}, I P L R_{i, I_{n}}$.

5) $S L S_{i, I_{n}} \rightarrow$ Sched determines the time interval $\left[t_{i, I_{n}, 0}, t_{i, I_{n}, f}\right]$ in which the service is due to be scheduled, where $t_{i, I_{n}, 0}$ expresses the SLS starting time and $t_{i, I_{n}, f}$ the SLS expiring time.

\section{Downstream SLSs}

In a domain $D_{x}$, when defining and negotiating an SLS with a downstream domain $D_{x}^{+}$, i.e., an $S L S_{i, E_{m}}^{+}$, the contracted service from an egress node $E_{m}$ should foresee the provision of adequate service levels taking into account all active $S L S_{i, I_{n}}$ going through $E_{m}$. From an $E_{m}$ perspective, specifying a downstream $S L S_{i, E_{m}}^{+}$follows the SLS template and notation introduced above for upstream SLSs, adding the sign +.

\section{Traffic Flows}

Depending on each application ability for signaling its service requirements, a traffic flow $F_{j}$ may undergo either implicit or explicit AC. For implicit AC, the relevant fields to consider include the source, destination and service class identifiers, i.e., $S r c_{i d}, D s t_{i d}, S C_{i d}$. For explicit AC, in addition to these fields, specifying a flow includes defining the traffic profile TProf, the required QoS parameters $\operatorname{Req} Q o S$ and an optional QoSTolerance. Their notation is similar to the one introduced for $S L S_{i, I_{n}}$.

\section{AC Criteria Specification}

The service-dependent AC criteria resort to (i) ratebased SLS control rules and (ii) QoS parameters control rules. These rules follow the notation introduced in Sec. III-B.

Rate-based SLS Control Rules - For each ingress node $I_{n} \in I^{D_{x}}$ and each egress node $E_{m} \in E^{D_{x}}$ one or more SLSs can be in place. As each $S L S_{i, I_{n}}$ and $S L S_{i, E_{m}}^{+}$ have specified a negotiated rate, $R_{i, I_{n}}$ and $R_{i, E_{m}}^{+}$respectively, a rate-based Measure-Sum algorithm can be applied to control SLSs utilization at each network edge node.

Explicit AC: At each ingress node $I_{n}$, verifying if a new flow $F_{j} \in S L S_{i, I_{n}}$ can be admitted involves testing if the $S L S_{i, I_{n}}$ can accommodate the new flow traffic profile, i.e.,

$$
\tilde{R}_{i,\left(I_{n}, *\right)}+r_{j} \leq \beta_{i, I_{n}} R_{i, I_{n}} .
$$

In (1), $\tilde{R}_{i,\left(I_{n}, *\right)}$ is the current measured rate of flows using $S L S_{i, I_{n}} ; r_{j}$ is the rate of the new flow $F_{j} ; 0<$ $\beta_{i, I_{n}} \leq 1$ is a safety margin defined for the negotiated rate $R_{i, I_{n}}$.

When the destination of flow $F_{j}$ is outside $D_{x}$, verifying if the new flow can be admitted also involves testing if the downstream $S L S_{i, E_{m}}^{+}$can accommodate the new flow traffic profile, i.e.,

$$
\tilde{R}_{i,\left(*, E_{m}\right)}^{+}+r_{j} \leq \beta_{i, E_{m}}^{+} R_{i, E_{m}}^{+} .
$$

In (2), $\tilde{R}_{i,\left(*, E_{m}\right)}^{+}$is the current measured rate of flows using $S L S_{i, E_{m}}^{+}$, considering all ingress-to- $E_{m}$ estimated rates of flows going through $E_{m}$, i.e.,

$$
\tilde{R}_{i,(*, E m)}^{+}=\sum_{k=1}^{N} \tilde{r}_{i,\left(I_{k}, E_{m}\right)} ;
$$

$r_{j}$ is the rate of the new flow $F_{j} ; 0<\beta_{i, E_{m}}^{+} \leq 1$ is the safety margin for the rate $R_{i, E_{m}}^{+}$defined in $S L S_{i, E_{m}}^{+}$. This safety margin determines the degree of overprovisioning for $S C_{i}$. The value of $\beta_{i, E_{m}}^{+}$may result from high-level domain policies defined at service class level, instead of being defined at SLS level.

The rate control rules for the admission of flows not sustained by an SLS, i.e., $F_{j} \notin S L S_{i, I_{n}}$, resort to (2) using the measured rate $R_{i, I_{n}}^{\notin S L S}$, i.e.,

$$
\tilde{R}_{i,\left(I_{n}, *\right)}^{\notin S L S}+r_{j} \leq \beta_{i, I_{n}}^{\notin} R_{i, I_{n}}^{\notin S L S} .
$$

Implicit $A C$ : For a service class $S C_{i}$ under implicit $\mathrm{AC}$, as flows are unable to describe $r_{j}$, the SLS control equations defined above become similar to the QoS control equation (see (5)), considering $P_{i, p}$ as a ratebased parameter. Therefore, traffic flows are accepted or rejected implicitly according to the value of a variable $A C_{-}$Status $_{\Delta t_{i}}$ computed once for $\Delta t_{i}$.

QoS Parameters Control Rules - When controlling the QoS levels in a domain, the QoS parameters and corresponding thresholds may vary depending on each service class $S C_{i}$ commitments, the statistical properties of the traffic and degree of overprovisioning. At each ingress node $I_{n}$, the $A C_{-} S_{\text {tatu }} s_{\Delta t_{i}}$ variable, used to control the admission of new flows in the monitoring interval $\Delta t_{i}$, is updated after checking the controlled parameters $P_{i, p}$ of $S C_{i}$, provided by egress nodes, against the corresponding pre-defined thresholds $T_{i, p}$, i.e.,

$$
\forall\left(P_{i, p}, \beta_{i, p}\right) \in P_{S C_{i}}: \tilde{P}_{i, p} \leq T_{i, p}
$$

where $\tilde{P}_{i, p}$ is the measured value of $P_{i, p}$ for $\Delta t_{i}$, and $T_{i, p}$ is the parameter's threshold, as explained in Sec. III-B. Equation (5) is not flow dependent, i.e., it is checked once during $\Delta t_{i}$ to determine AC_Status $_{\Delta t_{i}}$. The AC_Status $\Delta_{t_{i}}$ - accept - indicates that the measured QoS levels for $S C_{i}$ are in conformance with the QoS objectives and, therefore, new flows can be 
accepted. The AC_Status ${ }_{\Delta t_{i}}$ - reject - indicates that no more flows should be accepted until the class recovers and restores the QoS target values. This will only be checked at $\Delta t_{i+1}$.

\section{HANDLING CONCURRENCY}

A distributed AC model may involve multiple nodes making concurrent AC decisions. Therefore, dealing with concurrency is a key aspect to avoid over or false acceptance. This problematic is present whenever distributed and simultaneous AC decisions are taken, being more notorious on measurement-based approaches.

Focusing on the distributed AC model presented above, within a measurement time interval $\Delta t_{i}$, each ingress node $I_{n}$ makes AC decisions based on measures estimated for the interval, without knowing the contribution of other ingress nodes to the metrics variation until $\Delta t_{i+1}$, i.e., when the next measuring update takes place $^{2}$.

The presence of concurrency affects both the measured utilization of the rate related variables (e.g., $\tilde{R}_{i, E_{m}}^{+}$) shared among ingress nodes and the QoS measures. Note that, although these QoS measures reflect the available service between each $\left(I_{n}, E_{m}\right)$ pair, the links in the corresponding path may carry traffic resulting from a different pair of nodes. Therefore, the acceptance decisions at any other ingress node $I_{n^{\prime}} \neq I_{n}$ may affect the measured QoS for a specific $\left(I_{n}, E_{m}\right)$ pair. Regarding $\tilde{R}_{i, E_{m}}^{+}$, when a downstream $S L S_{i, E_{m}}^{+}$ is shared among all possible $\left(*, E_{m}\right)$ pairs (see (2)), the available rate of $S L S_{i, E_{m}}^{+}$in $\Delta t_{i}$ viewed by each $I_{n}$, i.e., $\beta_{i, E_{m}}^{+} R_{i, E_{m}}^{+}-\tilde{R}_{i,\left(*, E_{m}\right)}^{+}$, will change upon each ingress $I_{n}$ acceptance decision. Even if each $I_{n}$, individually, would update the measured rate $\tilde{R}_{i,\left(*, E_{m}\right)}^{+}$ according to new flow's accepted rate $\left(r_{j}\right)$, the side effect of other ingress admissions would be unknown until $\Delta t_{i+1}$. So, false or over acceptance could still occur during $\Delta t_{i}$.

The problem of mis-acceptance within each service class can be reduced resorting to larger safety margins $\left(\beta_{i, E_{m}}^{+}, \beta_{i, p}\right)$ to absorb the effect of traffic load fluctuations resulting both from the inherent statistical properties of traffic and from concurrent AC. Here, to reduce or solve the negative effects of concurrent AC might have on service offering, we explore and debate solutions such as: (A) the definition of a concurrency index based on the number of concurrent ingress nodes, affecting explicitly the rate control rules; (B) a token-based system to rule and limit the number of simultaneous AC decisions; (C) a rate-based credit

\footnotetext{
${ }^{2}$ In order to maintain simplicity, reduce overhead and latency associated with the exchange of control information, during $\Delta t_{i}$ each $I_{n}$ only knows (i) the initial measures provided by each $E_{m}$ for that time interval and (ii) its own contribution for the rate metric variation.
}

system to control each $I_{n}$ admission capacity. These solutions are not mutually exclusive as, for instance, a concurrency index may complement a token-based system. The following topics explore these scenarios, regarding the control of $S L S_{i, E_{m}}^{+}$utilization.

\section{Initial AC scenario}

In the case of explicit AC, we consider that satisfying the inequality $\tilde{R}_{i,\left(*, E_{m}\right)}^{+}+r_{j} \leq \beta_{i, E_{m}}^{+} R_{i, E_{m}}^{+}$determines a positive AC decision. When a new flow acceptance occurs, $\tilde{R}_{i,\left(*, E_{m}\right)}^{+}$can be updated by considering $r_{j}$ at the corresponding $I_{n}$, assuring that $I_{n}$ does not accept more traffic than the estimated available rate for $S L S_{i, E_{m}}^{+}$during $\Delta t_{i}{ }^{3}$. However, assuming that other concurrent ingress nodes are in place, the total new load is temporarily unknown and the available rate at $S L S_{i, E_{m}}^{+}$may be exceeded.

\section{A. Concurrency index}

Considering $\ddot{I}$ the set of concurrent ingress nodes sharing a common $S L S_{i, E_{m}}^{+}$, the estimation of $S L S_{i, E_{m}}^{+}$available rate for $\Delta t_{i}$ can be protected by a concurrency index $\chi_{i, E_{m}}$, which depends on the cardinality of $\ddot{I}$. In this way, explicit flow AC is ruled by

$$
r_{j} \leq \frac{\beta_{i, E_{m}}^{+} R_{i, E_{m}}^{+}-\tilde{R}_{i,\left(*, E_{m}\right)}^{+}}{\chi_{i, E_{m}}}
$$

where $\beta_{i, E_{m}}^{+} R_{i, E_{m}}^{+}-\tilde{R}_{i,\left(*, E_{m}\right)}^{+}$represents the estimated available rate of $S L S_{i, E_{m}}^{+}$to be shared among concurrent ingress nodes. In the case of implicit AC, a similar use of $\chi_{i, E_{m}}$ can be applied.

\section{B. Token-based system}

Other possible solution to control the number of concurrent ingress nodes performing AC decisions may follow a token-based system, where the level of concurrency allowed is determined by the number of tokens available.

In this system, only ingress nodes holding a token can accept new flows in $\Delta t_{i}$. In the limit, when a single token is available in the system, no concurrency is allowed. Nevertheless, if during $\Delta t_{i}$ the tokens pass through several ingress nodes, the $S L S_{i, E_{m}}^{+}$utilization can change without common knowledge of all concurrent nodes, $\ddot{I}$. Consequently, overacceptance may still occur. To cope with this, tokens can be used to carry $S L S_{i, E_{m}}^{+}$updates. If the token assignment remains

\footnotetext{
${ }^{3}$ Updating rate estimations leads to a more conservative $\mathrm{AC}$ as the rates of new flows are considered but the compensation effect of flows' departure is not taken into account. Keeping rate estimation at $I_{n}$ unchanged during $\Delta t_{i}$ explores this compensation effect but may increase over acceptance.
} 


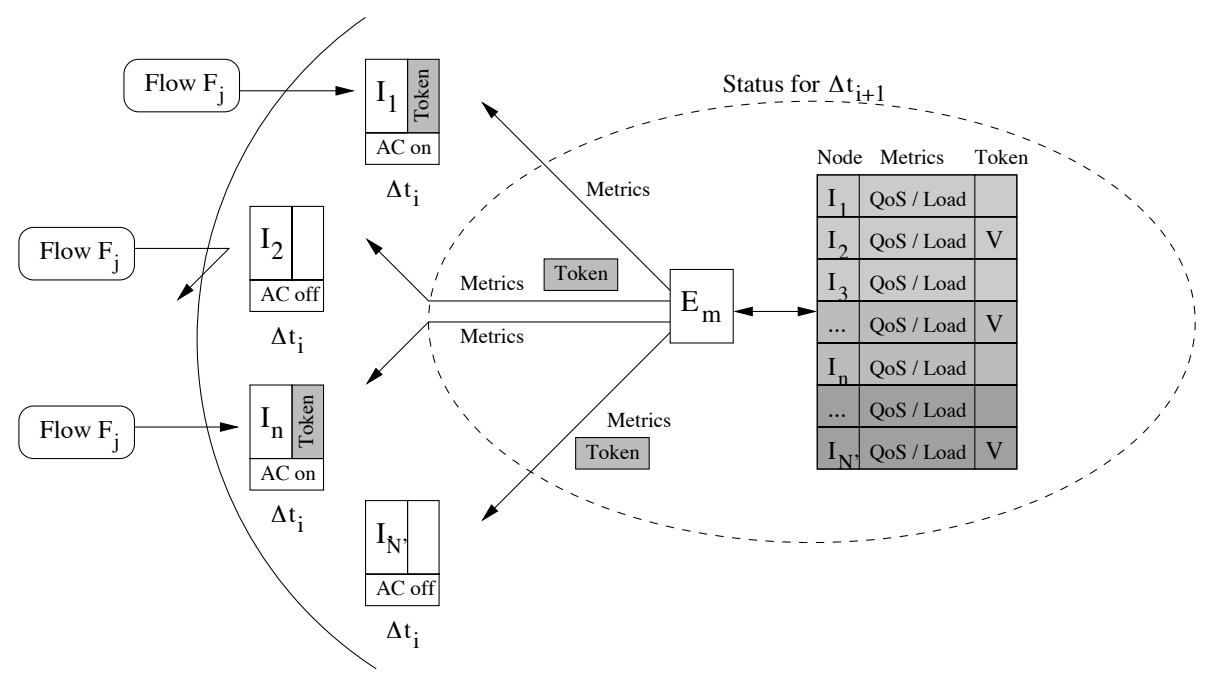

Fig. 3. Token-based system

unchanged during $\Delta t_{i}$, this time interval needs to be carefully defined as it influences directly the domain QoS stability and load balancing, and the AC latency at ingress nodes without tokens.

Apart from the conceptual simplicity of a tokenbased model to control concurrency, this method reduces the problem but does not solve it completely. Additionally, the signaling required for token exchange among ingress nodes and the time required for $I_{n}$ to get a token, which depends on the number of available tokens and the number of concurrent nodes $|\ddot{I}|$, may be prohibitive. To reduce this overhead, the number of available tokens and the choice of ingress nodes which might get one are controlled by the egress nodes based on their global view of each ingress rate usage. Topology information may also be used to group ingress nodes into topological independent areas according to the bottleneck link sharing [6]. Each defined group would share a single token or more tokens according to the degree of concurrency allowed. However, it should be noticed that a token based strategy may impair the admission of flows $F_{j} \in S L S_{i, I_{n}}$, i.e., a flow request not exceeding the available $S L S_{i, I_{n}}$ rate may be rejected or delayed by an $I_{n}$ not having a token. In this way, this strategy is more suitable to control the admission of concurrent $F_{j} \notin S L S_{i, I_{n}}$.

\section{Rate-based credit system}

To reduce the underlying drawbacks of a token-based system, the strategic view an egress node has of each measured rate $\tilde{r}_{i,\left(I_{n}, E_{m}\right)}$ can be used to implement a rate-based credit system to control the bandwidth usage of ingress nodes and, implicitly, concurrency. This system should respect the $I_{n}$ commitments previously assumed as regards the accepted $S L S_{i, I_{n}}$. Therefore, above all, this credit system should drive SLS AC and could also be explored to guide dynamic SLS AC decisions.

Following the defined AC model strategy, in the proposed rate-based credit system, the monitoring information obtained at egress node $E_{m}$ is used to control the amount of credits assigned to $I_{n}$, from an $\left(I_{n}, E_{m}\right)$ and service class $S C_{i}$ perspective. Each egress $E_{m}$ manages a pool of unused credits in order to distribute spare resources (bandwidth) dynamically as a complement to the static credit assignment initially defined, considering the ingress nodes grouped into distinct topological areas. The amount of available credits to be shared by ingress nodes that want to reach a specific egress $E_{m}$, therefore, controlled by this one, should consider:

- the network topology, the underlying bottleneck capacity $^{4}$ and network core multiplexing effects;

- the bandwidth sharing policies among classes [6];

- the already accepted $S L S_{i, I_{n}}$ and the corresponding expected traffic matrix;

- the $S L S_{i, E_{m}}^{+}$negotiated rate or the capacity allocated at $E_{m}$ for $S C_{i}$;

- internal policies regarding $R^{\in S L S}$ and $R^{\notin S L S}$ shares;

- a safety margin of unused credits at each $I_{n}$ to assure that $I_{n}$ has a controlled autonomy to make acceptance decisions during $\Delta t_{i}$.

At each $\Delta t_{i}$, ingress nodes may receive new credits

${ }^{4}$ Network bottleneck can be hard to define as it changes dynamically. Different pairs of $\left(I_{n}, E_{m}\right)$ may share and be limited in rate by a known bottleneck link; however, a new bottleneck may occur in a different place depending on traffic load and $\left(I_{n}, E_{m}\right)$ pairs involved. This concept is not new and is usually expressed by metrics such as: (i) available capacity and (ii) available bandwidth. For an initial credit assignment, (i) determines the bottleneck link between $\left(I_{n}, E_{m}\right)$. 


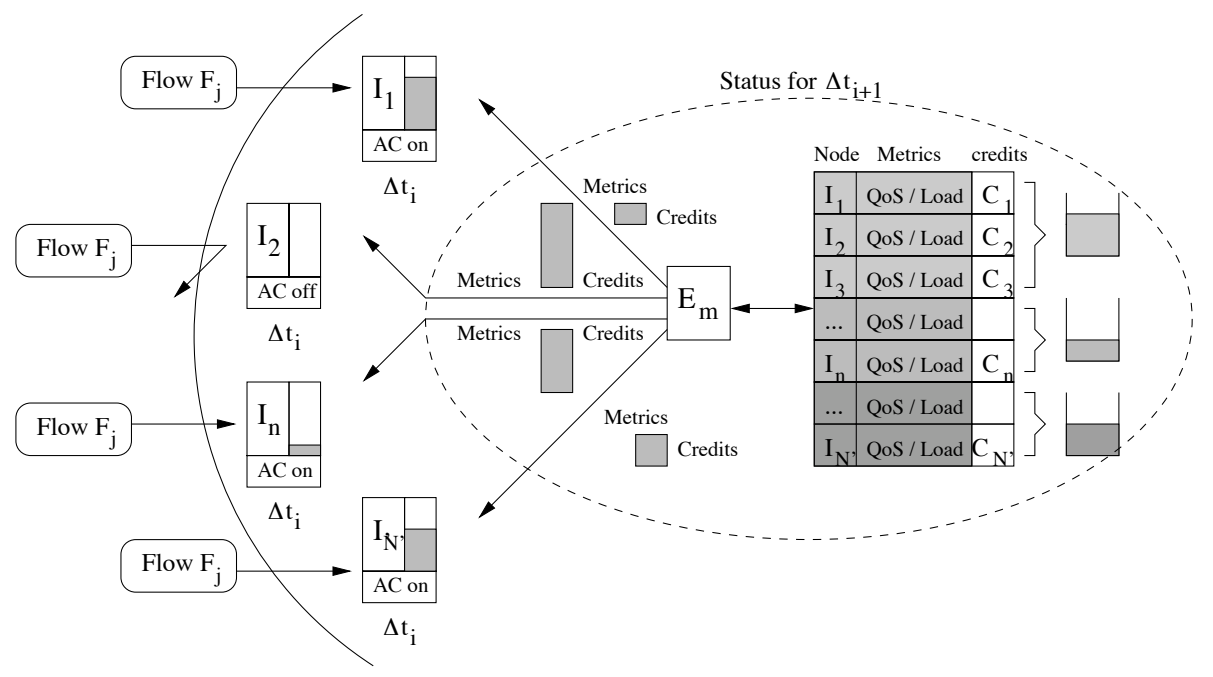

Fig. 4. Rate-based credit system

using the QoS metric dissemination process. When an egress node $E_{m}$ provides new measures to an ingress node $I_{n}$, it can distribute new credits too, i.e., no specific or additional control messages are needed (see Fig. 4). This strategy avoids several drawbacks of the solution proposed in [6], such as horizontal sharing of credits, use of specific signaling between ingress nodes and holding to many unused resources at each $I_{n}$.

The pool of unused credits at $E_{m}$ can either have one type of credits shared between $R^{\in S L S}$ and $R^{\notin S L S}$ or differentiated credits and bounds for the two cases. While both rates use $\left(I_{n}, E_{m}\right)$ path resources and indirectly interfere with QoS metrics, they can have a different weight in the global rate/credits usage. Distinguishing two types of credits assumes that $E_{m}$ can distinguish $I_{n}$ traffic as belonging or not to an $S L S_{i, I_{n}}$ when measuring $\tilde{r}_{i,\left(I_{n}, E_{m}\right)}$. To simplify the proposed credit system we can consider just one type of credits and let $I_{n}$ control the rate of each type of traffic applying pre-defined shares to the globally assigned credits. This would reduce the state information and avoid extra filters in $\tilde{r}_{i,\left(I_{n}, E_{m}\right)}$ estimation.

The management of credits can be either measurement-based or explicit, with credits being captured and released according to $S L S_{i, I_{n}}$ acceptance and termination. A possible measurement-based approach for managing the distribution of rate credits is detailed in Algorithm 1. In an explicit approach, two scenarios can be devised:

- each ingress node $I_{n}$ informs explicitly the egress node $E_{m}$ of the amount of credits captured or released, keeping the credits captive during the service scheduling period defined in the $S L S_{i, I_{n}}$;

- each egress $E_{m}$ uses the measured rate $\tilde{r}_{i,\left(I_{n}, E_{m}\right)}$ to determine when $I_{n}$ needs additional credits, waiting for an explicit teardown before releasing credits previously assigned. This avoids removing temporarily unused credits of SLSs still active, assuring that new incoming flows $F_{j} \in S L S_{i, I_{n}}$ have credits available.

At domain egress nodes, the amount of available credits in the pool may change for different reasons:

- credits are increased when: (i) the negotiated rate $R_{i, E_{m}}^{+}$and/or the links' capacity are upgraded; (ii) an $S L S_{i, I_{n}}$ having $E_{m}$ within its scope expires (explicit case, with $I_{n}$ returning credits back); (iii) the egress $E_{m}$ senses a rate utilization decrease at $I_{n}$, recovering excess credits (measurement-based case);

- the amount of available credits is decreased in favor of one $I_{n}$ when: (i) $I_{n}$ is running out of credits, i.e., its previous credit assignment is reaching an usage limit; this can be sensed by egress $E_{m}$ when measuring the rate $\tilde{r}_{i,\left(I_{n}, E_{m}\right)}$ or (ii) an explicit request occurs from $I_{n}{ }^{5}$.

\section{Decoupling AC Decisions}

Controlling the utilization of $R_{i, E_{m}}^{+}$during $\Delta t_{i}$ and, consequently, the concurrency control of $S L S_{i, E_{m}}^{+}$can be simplified if the AC module and corresponding tasks are divided between ingress and egress nodes. Instead of controlling the rates $R_{i, I_{n}}, R_{i, I_{n}}^{\notin S L S}$ and $R_{i, E_{m}}^{+}$at $I_{n}$, the control of $R_{i, E_{m}}^{+}$can be passed to $E_{m}$. For example, a flow request $F_{j} \in S L S_{i, I_{n}}$ crossing the domain $D_{x}$ is accepted at $I_{n}$ if Eqs. (1) and (5) are satisfied. When arriving at egress $E_{m}, F_{j}$ is accepted and may be

\footnotetext{
${ }^{5}$ Specific requests of credits from $I_{n}$ to $E_{m}$ during $\Delta t_{i}$ can also be considered, however, it changes the initial concept and assumption of viewing $\Delta t_{i}$ as a black-box, reflecting a measurement steady state.
} 


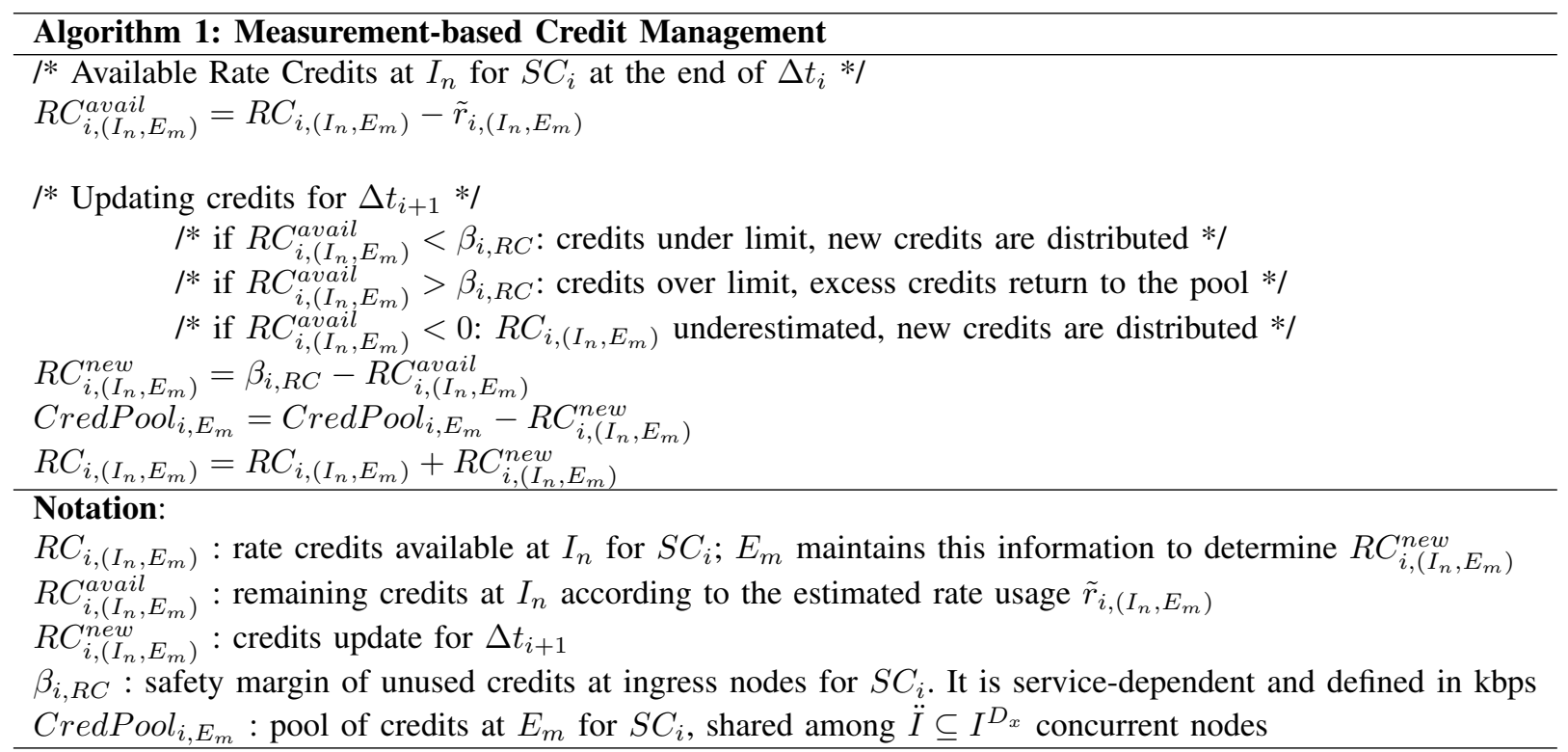

forward to the next domain if (2) is satisfied. When it is rejected at $E_{m}$, a reject notification due to $R_{i, E_{m}}^{+}$ underestimation or by an incorrectly defined statistical multiplexing factor may be reported.

Decoupling $\mathrm{AC}$ between $I_{n}$ and $E_{m}$ nodes, apart from being conceptually correct as $S L S_{i, I_{n}}$ is related to $I_{n}$ and $S L S_{i, E_{m}}^{+}$to $E_{m}$, brings other clear advantage. In fact, the overacceptance or concurrency control of $R_{i, E_{m}}^{+}$during $\Delta t_{i}$ becomes straightforward. Since each egress node $E_{m}$ can have a global view of all new flow requests trying to use $S L S_{i, E_{m}}^{+}$, for all $I_{n}$, it can update the previous $\tilde{R}_{i, E_{m}}^{+}$estimation in $\Delta t_{i}$ accordingly. This means that, when $E_{m}$ accepts a new flow it can update $\tilde{R}_{i, E_{m}}^{+}$to $\tilde{R}_{i, E_{m}}^{+}-r_{j}$, maintaining an updated view of global $S L S_{i, E_{m}}^{+}$occupancy. In this way, overacceptance as regards $R_{i, E_{m}}^{+}$cannot occur ${ }^{6}$. An obvious disadvantage is the additional computational burden of identifying and processing flow requests at each $E_{m}$.

Thus, the decision of decoupling AC decisions between $I_{n}$ and $E_{m}$, apart from the concurrency debate, should consider both (i) the computational overhead balance between $\left(I_{n}, E_{m}\right)$ QoS monitoring and $\mathrm{AC}$ tasks and (ii) the required state information at edge nodes.

When egress nodes perform $S L S_{i, E_{m}}^{+} \mathrm{AC}$, the credit strategy may still be useful to control each $I_{n}$ rate share, SLS AC, traffic entering $I_{n}$ not involving an $S L S_{i, E_{m}}^{+}$ and, indirectly, the QoS levels in the involved paths.

\section{Conclusions}

In this paper, we have addressed the issue of controlling concurrent $\mathrm{AC}$ in multiservice IP networks so that

${ }^{6}$ Note that when a flow is rejected at $E_{m}, \tilde{R}_{i, I_{n}}$ remains overestimated till $\Delta t_{i+1}$, as its rate $r_{j}$ is incorrectly accounted for. service level guarantees are protected from flows' overacceptance. This overacceptance results essentially from having multiple ingress nodes handling simultaneously multiple flow admission requests. We have pointed out simple solutions such as the use of a servicedependent concurrency index, a token-based or a ratebased system to reduce the chance of QoS violations that concurrent AC may raise. For the latter approach, a measurement-based credit management algorithm has also been proposed. Current work is focused on tuning and assessing the performance of these solutions considering a multiservice domain.

\section{REFERENCES}

[1] G. Huston. Next Steps for the IP QoS Architecture. RFC 2990 (Informational), November 2000.

[2] R. Atkinson, S. Floyd, and Internet Architecture Board. IAB Concerns and Recommendations Regarding Internet Research and Evolution. RFC 3869 (Informational), August 2004.

[3] J. Babiarz, K. Chan, and F. Baker. Configuration Guidelines for DiffServ Service Classes. draft-baker-diffserv-basic-classes04.txt, (work in progress), October 2004.

[4] Solange Rito Lima, Paulo Carvalho, and Vasco Freitas. Distributed Admission Control for QoS and SLS Management. Journal of Network and Systems Management - Special Issue on Distributed Management, 12(3):397-426, September 2004.

[5] Solange Rito Lima, Paulo Carvalho, and Vasco Freitas. Selfadaptive Distributed Management of QoS and SLSs in Multiservice Networks. In IEEE/IFIP International Conference on Integrated Management (IM 2005), Nice, France, May 2005. IEEE Press.

[6] A. Bak, W.Burakowski, F. Ricciato, S. Salsano, and H. Tarasiuk. Traffic Handling in AQUILA QoS IP Networks. In M. Smirnov, J. Crowcroft, J. Roberts, and F. Boavida, editors, QofIS'01, volume 2156, pages 243-260, September 2001.

[7] Z. Duan, Z. Zhang, Y. Hou, and L. Gao. A Core Stateless Bandwidth Broker Architecture for Scalable Support of Guaranteed Services. IEEE Transactions on Parallel and Distributed Systems, 15(2):167-182, February 2004. 
[8] B. Teitelbaum, S. Hares, L. Dunn, R. Neilson V. Narayan, and F. Reichmeyer. Internet2 QBone: building a testbed for differentiated services. IEEE Network, 13(5):8-16, "Sep./Oct." 1999.

[9] I. Stoica and Hui Zhang. Providing Guaranteed Services Without Per Flow Management. In ACM SIGCOMM'99, October 1999.

[10] L. Westberg. Resource Management in Diffserv (RMD) Framework. IETF draft: draft-westberg-rmd-framework-04.txt (work in progress), September 2003.

[11] S. Jamin, P. Danzig, S. Shenker, and L. Zhang. A MeasurementBased Call Admission Control Algorithm for Integrated Services Packet Networks (Extended Version). IEEE/ACM Transactions on Networking, pages 56-70, February 1997.

[12] L. Breslau and S. Jamin. Comments on the Performance of Measurement-Based Admission Control Algorithms. In IEEE INFOCOM'O0, March 2000.

[13] C. Cetinkaya, V. Kanodia, and E. Knightly. Scalable Services via Egress Admission Control. IEEE Transactions on Multime dia, 3(1):69-81, March 2001.

[14] V. Elek, G. Karlsson, and R. Rnngren. Admission Control Based on End-to-End Measurements. In IEEE INFOCOM'O0, 2000.

[15] L. Breslau, E. Knightly, S. Shenker, I. Stoica, and H. Zhang. Endpoint Admission Control: Architectural Issues and Performance. In ACM SIGCOMM'O0, 2000.

[16] R. Mortier, I. Pratt, C. Clark, and S. Crosby. Implicit Admission Control. IEEE Journal on Selected Areas in Communication, 18(12):2629-2639, December 2000.

[17] S. Fredj, S. Oueslati-Boulahia, and J. Roberts. Measurementbased Admission Control for Elastic Traffic. In 17th International Teletraffic Congress, June 2001.
[18] P. Morand, M. Boucadair, P. Levis, R. Egan, H. Asgari, D. Griffin, J. Griem, J. Spencer, P. Trimintzios, M. Howarth, N. Wang, P. Flegkas, K. Ho, S. Georgoulas, G. Pavlou, P. Georgatsos, and T. Damilatis. Mescal D1.2 - Initial Specification of Protocols and Algorithms for Inter-domain SLS Management and Traffic Engineering for QoS-based IP Service Delivery and their Test Requirements. Mescal Project IST-2001-37961, January 2004.

Solange Rito Lima graduated in 1991, received her MSc and PhD degrees in Computer Communications from the University of Minho, Braga, Portugal, in 1997 and 2006, respectively. She is currently an Assistant Professor in Computer Communications at the same University. Her research interests include multiservice IP networks and protocols, QoS, traffic control and monitoring issues.

Paulo Carvalho graduated in 1991 and received his $\mathrm{PhD}$ degree in Computer Science from the University of Kent at Canterbury, United Kingdom, in 1997. He is currently Assistant Professor of Computer Communications, Department of Informatics, at the University of Minho, Portugal. His main research interests include broadband technologies, multiservice networks and protocols, and mobile networks.

Vasco Freitas graduated in 1972 and obtained his MSc and $\mathrm{PhD}$ degrees in Control and Computer Communications at the University of Manchester, UK, in 1977 and 1980. From 1989 until 1994 he was Director of Networking at the Portuguese Foundation for Scientific Computing to establish the National University Data Network. He is currently Professor of Computer Communications at the University of Minho, Portugal. 